\title{
A!
}

This is an electronic reprint of the original article.

This reprint may differ from the original in pagination and typographic detail.

Tayyab, Muhammad; Koudouridis, G. P.; Gelabert, X.; Jantti, R.

\section{Handover Performance and Power Consumption Analysis of LTE Mobile Relays}

Published in:

2020 IEEE 92nd Vehicular Technology Conference, VTC 2020-Fall - Proceedings

DOI:

10.1109/VTC2020-Fall49728.2020.9348720

Published: 01/11/2020

Document Version

Peer reviewed version

Please cite the original version:

Tayyab, M., Koudouridis, G. P., Gelabert, X., \& Jantti, R. (2020). Handover Performance and Power

Consumption Analysis of LTE Mobile Relays. In 2020 IEEE 92nd Vehicular Technology Conference, VTC 2020-

Fall - Proceedings [9348720] (IEEE Vehicular Technology Conference). IEEE. https://doi.org/10.1109/VTC2020-

Fall49728.2020.9348720

This material is protected by copyright and other intellectual property rights, and duplication or sale of all or part of any of the repository collections is not permitted, except that material may be duplicated by you for your research use or educational purposes in electronic or print form. You must obtain permission for any other use. Electronic or print copies may not be offered, whether for sale or otherwise to anyone who is not an authorised user. 


\section{Handover Performance and Power Consumption Analysis of LTE Mobile Relays}

\author{
M. Tayyab ${ }^{1,3}$ \\ ${ }^{1}$ Huawei Technologies Finland Oy \\ Helsinki, Finland \\ muhammad.tayyab5@huawei.com \\ muhammad.tayyab@aalto.fi
}

\author{
G. P. Koudouridis ${ }^{2}$ and X. Gelabert ${ }^{2}$ \\ ${ }^{2}$ Huawei Technologies Sweden AB, \\ Kista, Sweden \\ george.koudouridis@huawei.com, \\ xavier.gelabert@huawei.com
}

\author{
R. Jäntti ${ }^{3}$ \\ ${ }^{3}$ Department of Communications and \\ Networking, School of Electrical \\ Engineering, Aalto University, Espoo, \\ Finland \\ riku.jantti@aalto.fi
}

\begin{abstract}
Mobile relay node (MRN) is one of the cheaper options for reliable communication when users are moving by public transport (i.e. bus, tram, train, subway, etc.), especially in urban areas. Critically, MRNs need to maintain a backhaul connection with the fixed infrastructure via a donor eNB, (DeNB). If the MRN fails to successfully handover (HO) from one DeNB to another, it will create a single point of failure, i.e. the connection of all UEs connected to MRN will be dropped. In this paper, we address the $\mathrm{HO}$ performance of a MRN including a power consumption analysis thereof. We investigate the potential gains in terms of HO rate, HO failure ratio (HOFR), ping-pong (PP) rate and power consumption (both at $\mathrm{UE}$ and $\mathrm{eNB}$ ) when a MRN is deployed on a bus traveling along the cell edges of surrounding macro BSs. We also look over the MRN HO failure cases to identify the causes of $\mathrm{HO}$ failures that are more critical for the UEs onboard. Numerical results indicate that deploying a MRN on the roof-top of a bus improves the HO rates $15 \%$, HOFR $8 \%$, PP rate $17 \%$, UE power consumption $21 \%$, and eNB power consumption $14 \%$ on average for all simulated cases. We have also established that UL transmission errors are the most dominant causes of turning MRN to a single point of failure during a $\mathrm{HO}$.
\end{abstract}

Keywords-Mobile relay node (MRN), LTE, handover, simulation, performance evaluation, power consumption.

\section{INTRODUCTION}

Reliable mobile communications with enhanced data rates are desirable when the users are moving with a moderate to high speed on public transport (i.e. bus, tram, train, etc.). This is because the onboard passengers would like to keep in contact with the outside world and normally utilize their time by reading online books, accessing emails, browsing the internet, playing online games and watching online videos, etc. [1]. With the rapid development in public transportation, more travelers choose this option. However, providing cellular services to onboard passengers is challenging due to high penetration loss, severe Doppler shift, reduced handover (HO) success rate, high user equipment (UE) power consumption and reduced spectral efficiency [2][3]. The high penetration loss for the onboard passengers is due to well-shielded public transportation with coated windows. If an onboard UE connects to the macro base station (BS), the penetration loss leads to low signal quality and thus low data rates. Also, the Doppler shift might occur because of the relative movement of the UE and the macro BS depending upon the speed of the motion that leads to worse channel conditions and even radio link failure (RLF). The Doppler Effect degrades the data demodulation accuracy due to the data symbol phase rotation. A high penetration loss and the Doppler shift limit

This project has received funding from the European Union's H2020 research and innovation program under grant agreement H2020-MCSA-ITN2016-SECRET 722424 [14] the cell coverage and lead to longer paging, cell (re-)selection, and $\mathrm{HO}$ procedure. It would be not uncommon to have 300 active UEs per train and 50 active UEs per bus [4]. Such a large amount of HOs would cause channel congestion due to increased signaling overhead, leading to high $\mathrm{HO}$ failures [4]. The major concern is the $\mathrm{HO}$ process, as the $\mathrm{HO}$ rate increases with speed [5]. If the time taken to cross the $\mathrm{HO}$ area is less than the minimum $\mathrm{HO}$ delay, then the $\mathrm{HO}$ process may fail which in turn causes service interruption [1]. In addition, a high UE power consumption would be expected due to the continuous measurements in both active and idle mode in a high mobility scenario.

Mobile Relay Nodes (MRNs) are used to improve the spectral efficiency by reducing the "infrastructure-to-user" distance and to extend the cell coverage. MRNs are a good candidate in a fast-moving environment and cheaper than existing solutions requiring dedicated eNBs, especially in urban areas [3]. The MRN has been extensively discussed in the 3rd Generation Partnership Project (3GPP) Release 12 (R12) to support cellular services in a fast-moving environment [3]. Therein, the study item on MRN focuses on performance evaluation of potential solutions and a comparison of different architecture choices implementing the MRN paradigm. The basic idea is to install MRNs on public vehicles. As the vehicles move, the MRN moves with the UEs within the carriage. The UEs onboard communicate with the MRN through an indirect access link and, in turn, the MRN communicates with the donor eNB (DeNB) using the backhaul link as shown in Fig. 1. The outboard UEs communicate with the eNB through a direct access link. The relative position of the onboard UEs with respect to the MRN is stationary or at pedestrian speed, ensuring good channel conditions and thus high data rates for this link [2].

In this paper, we provide an analysis of the HO performance in the presence of a MRN and the associated power consumption in LTE based on a scenario where a cluster of UEs is traveling on a bus. The bus moves along the cell edges of the macro BSs at speeds pertinent to those of an urban city environment. This scenario can be easily extended to trains, tramways, and private cars. Herein, we investigate the improvements in $\mathrm{HO}$ rate, HOFR, PP rate, along with UE and eNB power consumption when the MRN is located on the roof-top of a bus. We also identify the causes of MRN HO failures towards the DeNBs that will lead to a single point of failure for onboard UEs. Noteworthy, the performance of the MRN HO while taking into account the power consumption of the air-interface signaling is largely overlooked in the literature. This study addresses this issue and contributes to determining the potential benefits of using MRN and realizing the problem of MRN HO to the DeNB.

The rest of the paper is organized as follows: Section II provides an overview of the MRN HO mechanism in LTE. Section III discusses simulator modeling aspects. In Section IV, 


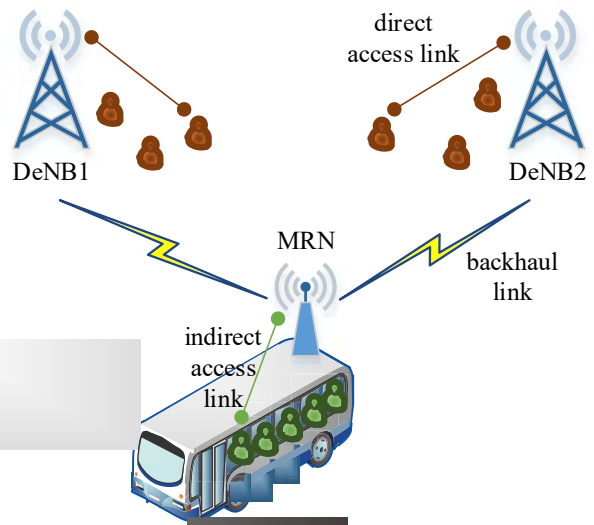

Fig. 1. MRN scenario.

numerical results are presented. And finally, Section V provides a conclusion with future research directions.

\section{OVERVIEW OF HANDOVER MECHANISM IN LTE}

Four equivalent MRN architecture alternatives (Alt.1 to Alt.4) have been discussed in 3GPP [3] of which Alt.1 is identical to the procedure for UE Inter-eNB mobility defined in 3GPP [7]. The HO procedure in architecture Alt 1 introduces no additional signaling for the UE HO in both radio access and core network side during MRN mobility. As such, it is the simplest and it demonstrates better latency performance [6], which justifies its selection for this study. The overall MRN HO procedure based on architecture Alt 1 is shown in Fig. 2 in an LTE network [3]. According to architecture Alt. 1, the MRN is connected to the DeNB via the Un interface while the UEs are connected to the MRN via the Uu interface.

The MRN measurement phase starts with the measurement of downlink (DL) reference signals (RS) from both the serving DeNB (s-DeNB) and neighboring DeNBs, including the potential target DeNB (t-DeNB), which is the DeNB "taking over" the handovered MRN. The MRN carries out signal strength (SS) measurements over a set of specific RS sent by the serving donor cell (s-Dcell) as well as the neighboring Dcells, and it computes the RS received power (RSRP) from each cell. After processing the measurements, if an "entry condition" is fulfilled, a measurement report (MeasReport) containing RSRP information and a candidate Dcell-list is transmitted by the MRN and received at the s-DeNB. The "A3 event" [7] is used as entry condition to assess if the RSRP of the t-DeNB is stronger than the RSRP of the s-DeNB plus a hysteresis margin (called A3 offset). To trigger the MeasReport, the entry condition has to be valid during a specified time defined by the time to trigger (TTT) parameter [7]. Once the MeasReport is correctly received at the $\mathrm{s}$-DeNB, the HO preparation phase between t-DeNB and $\mathrm{s}-$ DeNB starts and a HO request is issued from the s-DeNB to the $\mathrm{t}$-DeNB. The t-DeNB then decides whether or not it can admit the MRN and feedbacks this information to the s-DeNB. Upon successful admission, the $\mathrm{HO}$ execution phase starts when the sDeNB transmits the $\mathrm{HO}$ command (HOcmd) to the MRN with the necessary information to synchronize and perform initial access to the t-DeNB. Upon successful reception of the HOcmd, the MRN accesses the t-eNB, by means of a Random Access (RA) procedure via the RA Channel (RACH). With successful RA completion, the $t-D e N B$ receives a $\mathrm{HO}$ confirmation (HOconf) message from the MRN. Each of these steps contributes towards the overall signaling cost and latency to execute the HO. Hence, enhancing and optimizing these phases will facilitate the improvement of QoS and quality of experience (QoE) of the user/device.

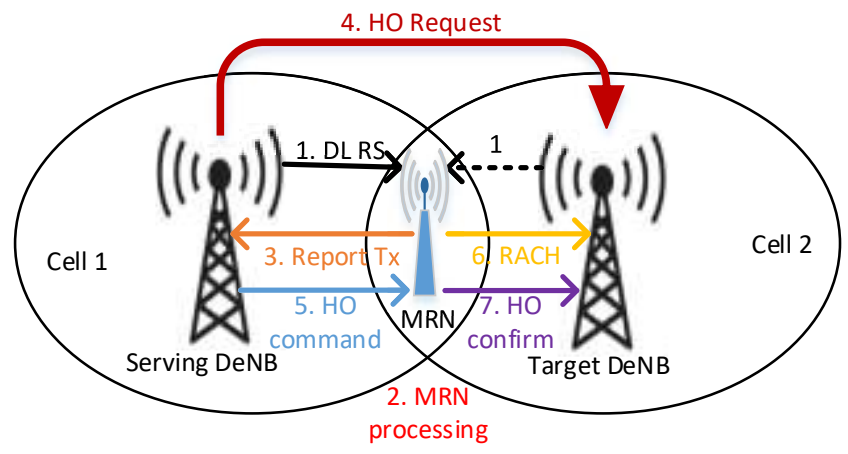

Fig. 2. MRN HO procedure (adapted from [3]) based on architecture Alt 1.

\section{SYSTEM MODEL}

A MATLAB based LTE system level simulator is used considering a hexagonal grid deployment of 16 tri-sectored eNBs with cell wrap-around to allow fair interference conditions across the scenario. A set of 200 UEs is randomly placed over the scenario, with the assumption that the first twenty-four UEs $(12 \%)$ are onboard at a fixed speed and a specified direction and the remaining $88 \%$ outboard UEs follow rectilinear motion at a fixed speed with initial random directions uniformly distributed between $\left[0^{\circ}, 360^{\circ}\right.$. The outboard UEs are randomly deployed outside the bus and all over the scenario. A roof-top mounted MRN is deployed on the bus. The users on the bus are getting cellular services from the MRN and the MRN is connected to a DeNB via a wireless backhaul. This wireless backhaul operates over the same bandwidth as the access link (i.e. in-band wireless backhaul is assumed). It is assumed that the bus is traveling along a road situated at the cell edge of the DeNBs across the simulation scenario. We motivate this by noting that DeNBs and MRN share the same access band and, therefore, MRN passing close to the DeNB may cause excessive interference and reduce the MRN cell coverage area. In addition, the UEs will experience poor radio link conditions at the cell edge. These UEs will then benefit from MRN following trajectory along DeNBs cell edges. The backhaul link between MRN and the DeNB is implemented in the simulator as shown in Fig. 1 to figure out the single point of failure for the MRN connected UEs. It is to be noted that the MRN follows the HO procedure the same as the UE HO procedure in an LTE network.

The simulation scenario which is shown in Fig. 3 has been based on the simulator we developed and thoroughly described in [5]. The main simulation assumptions covering the $\mathrm{HO}$ and power consumption models are summarized in Table I. The supplied power to the eNB, necessary to either transmit or receive signaling $S$ is denoted by $P_{e N B, S u p}^{S, T x / R x}$, and can be calculated as follows [11][12],

$$
P_{e N B, \text { sup }}^{S, T x / R x}=P_{e N B, T x / R x}^{S} / \eta+N_{T B}^{S} / N_{T B}^{D L} \cdot\left(P_{R F, e N B}+P_{B B}^{\prime}\right),
$$

where $P_{e N B, T x / R x}^{S}$ is the allocated eNB transmitted or received power (in W) per signaling message $s$ and $\eta$ is the power amplifier efficiency. $P_{R F, e N B}$ denotes the supply power contribution of the RF equipment, which is conveniently scaled by the portion of utilized resources by signaling message $S$. Similarly, $P_{B B}^{\prime}$ is the basic baseband unit (BBU) consumption in watts (see Table I). Equally, the supply power required for the UE to transmit or receive signaling message, $s$ is denoted by $P_{U E, \text { Sup }}^{S, T x / R x}$ and is given by [11][12], 


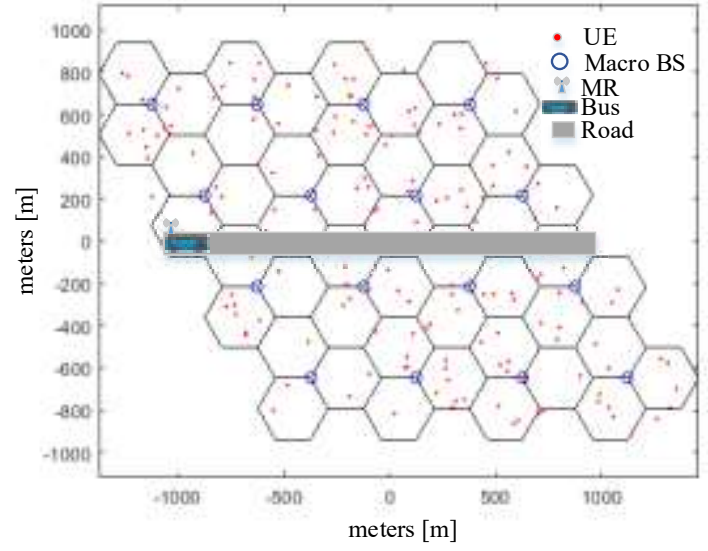

Fig. 3. The considered simulation scenario, with the bus following a wraparound trajectory over the specified road once it hits the rightmost border.

Table I Simulation parameters and assumptions.

\begin{tabular}{|c|c|}
\hline Feature & Implementation \\
\hline Network topology & $\begin{array}{c}\text { A hexagonal grid of } 16 \times 3=48 \text { cells (wrap-around } \\
\text { included) }\end{array}$ \\
\hline Inter-site distance & From the set $\{500,750,1000,1250\} \mathrm{m}$ \\
\hline System Bandwidth & $\begin{array}{c}B_{s y s}=5 \mathrm{MHz} \text { (paired FDD), with } N_{R B}^{D L}=N_{R B}^{U L}=25 \\
\text { RBs at carrier frequency } f_{c}=2.1 \mathrm{GHz}, 1 \mathrm{~TB}=6 \mathrm{RBs}, \\
\qquad N_{T B}^{D L}=N_{T B}^{U L}=|25 / 6|\end{array}$ \\
\hline eNB DL power & $43 \mathrm{dBm}$ \\
\hline MRN power & $27 \mathrm{dBm}$ \\
\hline UE Power & $23 \mathrm{dBm}$ \\
\hline Antenna patterns & 3D model specified in [8], Table A.2.1.1.2-2 \\
\hline Channel model & 6 tap model, Typical Urban (TU) \\
\hline Shadowing & $\begin{array}{c}\text { Log-normal shadowing: Mean } 0 \mathrm{~dB} \text {, Standard } \\
\text { deviation: } 8 \mathrm{~dB}\end{array}$ \\
\hline Propagation model & $\begin{array}{l}L=130.5+36.7 \log _{10}(R), R \text { in } \mathrm{km}, \text { for eNB } \\
L=140.7+36.7 \log _{10}(R), R \text { in } \mathrm{km}, \text { for MRN }[8]\end{array}$ \\
\hline UE and Bus speed & from the set $\{30,60\} \mathrm{km} / \mathrm{h}$ \\
\hline $\begin{array}{l}\text { RLF detection by L1 } \\
\text { of UE }\end{array}$ & $\begin{array}{l}\text { T310 }=1 \mathrm{~s}, \mathrm{~N} 310=1, \mathrm{~N} 311=1 \text { as specified in [9] } \\
\mathrm{Q}_{\text {in }}=-4.8 \mathrm{~dB} ; \mathrm{Q}_{\text {out }}=-7.2 \mathrm{~dB} \text { as specified in [10] }\end{array}$ \\
\hline HO parameters & $\mathrm{TTT}=\{64\} \mathrm{ms}, \mathrm{A} 3$ offset $=\{3\} \mathrm{dB}$. \\
\hline $\begin{array}{c}\text { Number of TBs per } \\
\text { each signaling message }\end{array}$ & $\begin{array}{c}N_{T B}^{M R}=1 \mathrm{~TB} ; N_{T B}^{H O c n f}=1 \mathrm{~TB} ; N_{T B}^{H O c m d}=2 \mathrm{TBs} \\
N_{T B}^{R A}=1 \mathrm{~TB} .[12]\end{array}$ \\
\hline $\begin{array}{c}\text { Power consumption } \\
\text { calculation parameters }\end{array}$ & $\begin{array}{c}\eta=0.311(31.1 \%), P_{R F, e N B}=12.9 \mathrm{~W} \\
P_{R F, U E}=2.35 \mathrm{~W}, P_{B B}^{\prime}=29.4 \mathrm{~W} \\
P_{T X B B}=0.62 \mathrm{~mW}, P_{R x B B}=0.97 . R_{R x}+8.16(\mathrm{~mW}) \\
{[12]}\end{array}$ \\
\hline $\begin{array}{l}\text { Signaling transmission } \\
\text { times }\end{array}$ & $\begin{array}{c}T_{e N B}^{H O c m d}=1 \mathrm{~ms} ; T_{U E}^{M R}=1 \mathrm{~ms} ; T_{U E}^{H O c n f}=1 \mathrm{~ms} \\
T_{U E}^{R A C H t x}=1 \mathrm{~ms}[12]\end{array}$ \\
\hline
\end{tabular}

$P_{U E, \text { sup }}^{S, T x / R x}=P_{U E, T x / R x}^{S}+N_{T B}^{S} / N_{T B}^{U L} \cdot\left(P_{R F, U E}+P_{T x / R x B B}\right)$,

where $P_{U E, T x / R x}^{S}$ is the allocated UE transmitted or received power (in W) per signaling message $s$, and where the supply power contribution to the RF and $\mathrm{BB}$ part is also scaled by the portion of utilized resources by signaling $S . P_{T \times / R \times B B}$ is the transmitted or received UE BBU power (see Table I) where $R_{R x}$ is the received data rate that is a multiplication of signaling rate and the carried bits in a transport block (TB). The time-averaged supply power to capture the time-domain system dynamics is given by,

$\bar{P}_{x, \text { sup }}^{s, T x / R x}=P_{x, \text { sup }}^{s, T x / R x} \cdot T_{x}^{s} \cdot R_{x}^{s}$,

where $T_{x}^{s}$ is the signaling duration in seconds and $R_{x}^{S}$ is the signaling rate which will be obtained from system-level simulations.

\section{NUMERICAL RESULTS AND DISCUSSION}

In this section, we provide a numerical evaluation of the $\mathrm{HO}$ performance and the total UE and eNB power consumption, with/without deploying a MRN at the roof-top of the bus. The $12 \%$ of the UEs are traveling on the bus and the $88 \%$ outboard UEs are moving outside the bus, all over the scenario. Two speed values are simulated where the speed $30 \mathrm{~km} / \mathrm{h}$ is presented with a "solid line" and speed $60 \mathrm{~km} / \mathrm{h}$ is shown with a "dashed line" in the following figures. Two following case scenarios are simulated to have a fair comparison,

- Case 1 is without MRN deployment on the bus meaning that all onboard UEs will perform their individual HO procedure with macro eNB.

- Case 2 is the same as Case 1 but with MRN, meaning that instead of all onboard UEs perform the individual $\mathrm{HO}$ procedure, only the MRN will perform the HO procedure to the DeNBs.

The impact of deploying a MRN on the HO rate is shown in Fig. 4. It is clear from the graph that Case 2 has a significant improvement in terms of $\mathrm{HO}$ rate, a $15 \%$ reduction in $\mathrm{HO}$ rate on average for all simulated cases. At speed $30 \mathrm{~km} / \mathrm{h}$, high improvement in $\mathrm{HO}$ rates is observed at high ISDs, almost double than the low ISDs while this improvement is almost constant at speed $60 \mathrm{~km} / \mathrm{h}$ for all ISDs. This is because, at high ISDs, the UEs have the poor radio link conditions at the cell edge, as a result, they suffer from high ping-pong (PP) rates at low speed that further leads to high $\mathrm{HO}$ rates.

The impact of deploying a MRN on the $\mathrm{HO}$ failure ratio (HOFR) is shown in Fig. 5. As per our assumption, when the MRN fails to $\mathrm{HO}$ to a t-DeNB, this increases the service interruption time for the onboard UEs until the MRN connects to another t-DeNB. We do not count the onboard UEs as also being in a $\mathrm{HO}$ failure. Overall, the HOF cases have been reduced for Case 2 except at $30 \mathrm{~km} / \mathrm{h}$ speed with high ISDs. The MRN may face poor radio link conditions at high ISDs and thus unable to connect to the DeNB. This creates a single point of failure for the UEs onboard. Now the UEs have to wait till the MRN gets connected to another DeNB and this increases the service interruption time for the onboard UEs. On the contrary, high speed helps the MRN to escape from the poor radio link condition area, so the gain in terms of reduced HOFR can be seen at $60 \mathrm{~km} / \mathrm{h}$. Overall, an $8 \%$ reduction in HOFR on average is observed for all simulated cases of Case 2.

Similarly, an improvement in the PP rate for Case 2 can be seen in Fig. 6, where the improvement at $60 \mathrm{~km} / \mathrm{h}$ speed is constant while the improvement at high ISDs cases of the 30 $\mathrm{km} / \mathrm{h}$ speed is high. The high PP rate at the low-speed and high ISDs, for Case 1 is due to the bad radio link conditions of the onboard UEs traveling at the cell edge of the macro eNBs. Significant improvements in terms of PP rate can be seen for Case 2 as the MRN improves the radio link conditions of the onboard UEs. This justifies the trends of high improvements in $\mathrm{HO}$ rates for $30 \mathrm{~km} / \mathrm{h}$ speed at high ISDs in Fig. 4. Overall, a $17 \%$ reduction in PP rate on average is noticed for all simulated cases of Case 2.

The total power consumption, which is the addition of both transmitted and received power consumption at the UE or eNB side, is calculated using (3). A total UE power consumption at various ISDs and speed values is shown in Fig. 7 and the total eNB power consumption is displayed in Fig. 8. The overall trend of the graphs shows that power consumption is reduced significantly for Case 2 . High power consumption can be seen in both graphs for Case 1 at $30 \mathrm{~km} / \mathrm{h}$ speed and high ISDs. This 


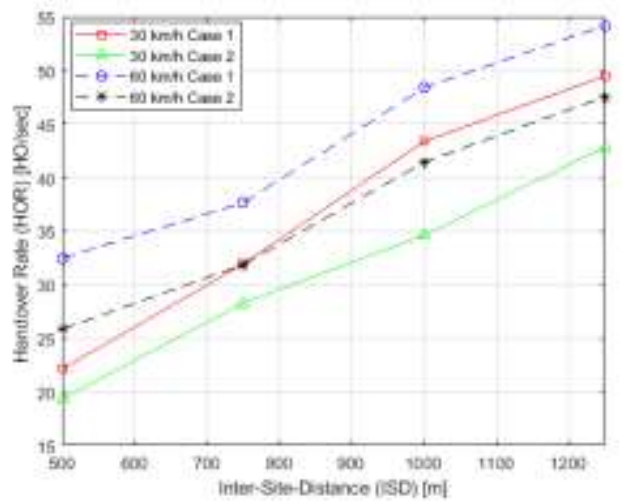

Fig. 4. Impact of deploying MRN on the HO rate.

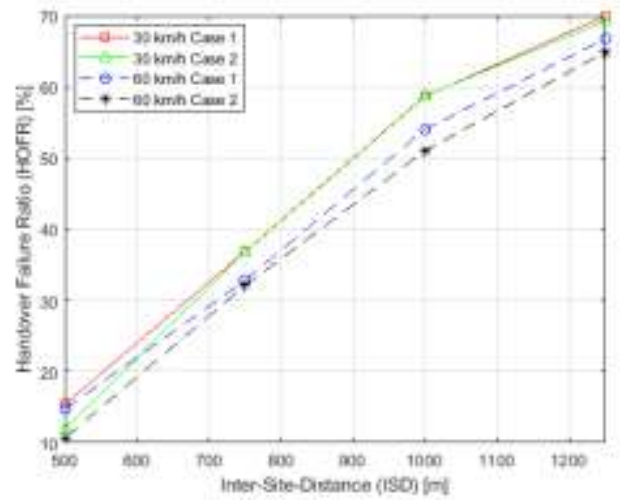

Fig. 5. Impact of deploying MRN on the $\mathrm{HO}$ failure ratio.

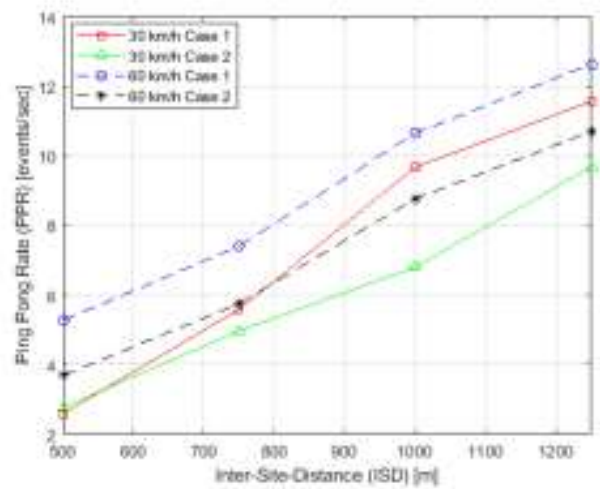

Fig. 6. Impact of deploying MRN on the PP rate.

is because of high signaling messages transmitted and received which is attributed to the high PP rate for this specific case that we noted in Fig. 6. Overall, a $21 \%$ reduction in UE and a $14 \%$ reduction in eNB power consumption on average is seen for all simulated cases of Case 2.

\section{A. Mobile Relay Node Handover Performance}

This subsection provides the performance of the MRN to figure out the single point of failure cases during the entire simulation. The HOFR breakdown per type (F0 to F7) of the MRN is shown in Fig. 9. Overall, the failure cases concentrate on the UL transmission errors (max. retransmission /RLC timer expires). Random access channel (RACH) failure is common at high ISD due to the MRN poor UL radio conditions close to the cell borders in large cells. At the ISD $500 \mathrm{~m}$ case, a high failure ratio is observed for low speed. This indicates that the low speed of the bus is not enough to escape the MRN from the poor

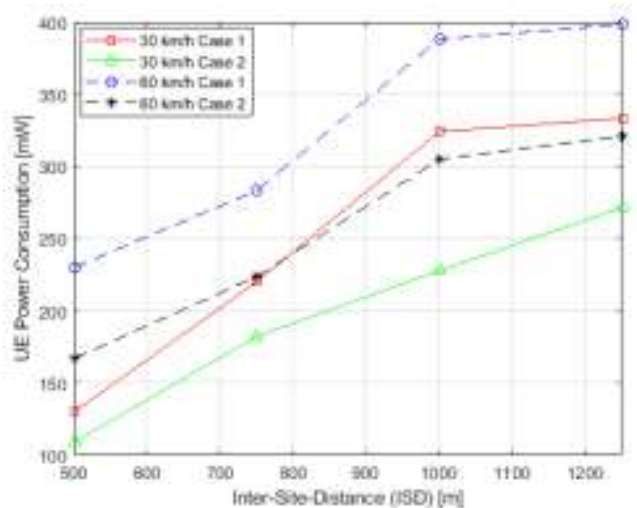

Fig. 7. Impact of deploying MRN on UE total power consumption.

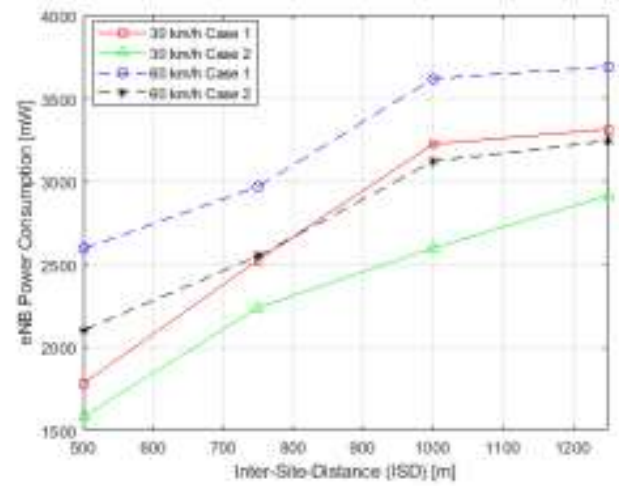

Fig. 8. Impact of deploying MRN on eNB total power consumption.

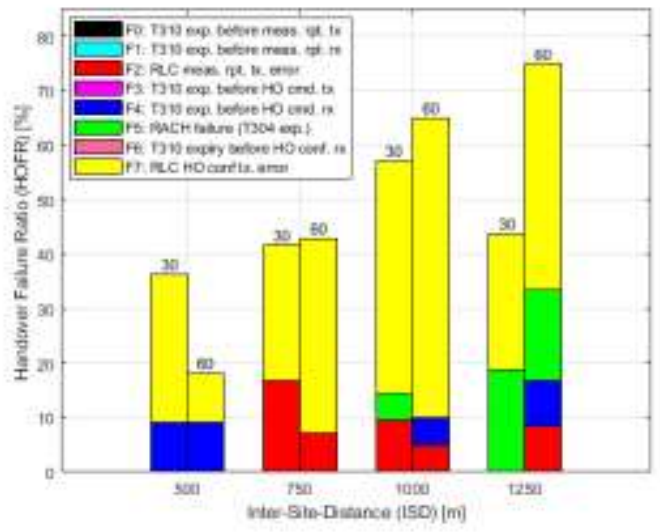

Fig. 9. Impact of ISD and UE speed on MRN HOF ratio breakdown per type.

condition areas. F4 failure type (timer T310 expiry before HOcmd reception) is due to the reason that the MRN move out of the s-DeNB and thus the HO command sent by the s-DeNB cannot reach to the MRN. Such types of failure create a single point of failure for the MRN connected UEs and this increases the service interruption time for the onboard UEs. This means that we can further improve the overall system $\mathrm{HO}$ performance metrics by reducing the MRN HO failure cases to the DeNB. As per Fig. 9, the ISD $500 \mathrm{~m}$ and speed $60 \mathrm{~km} / \mathrm{h}$ case is optimum for the MRN out of the simulated cases in terms of the lower single point of failures. The HO interruption time (HOIT) is the time period during the $\mathrm{HO}$ procedure when the MRN cannot exchange user plane packets with any of the DeNB [13]. During this time period, the onboard UEs will face service interruption. We found that the HOIT for the onboard passengers is reduced 


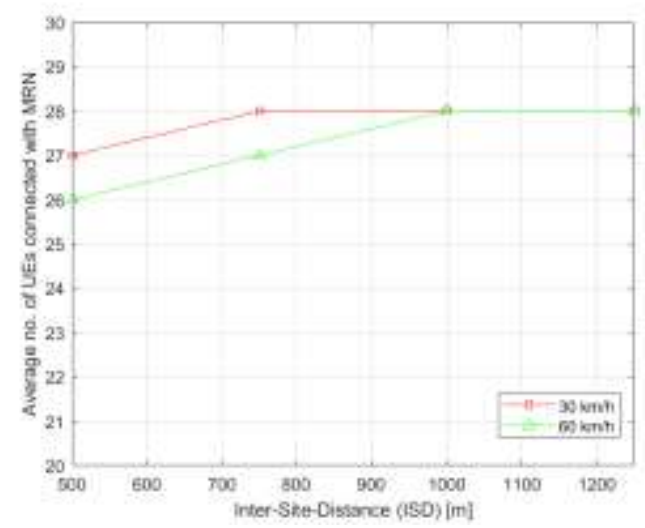

Fig. 10. The average number of UEs connected with MRN.

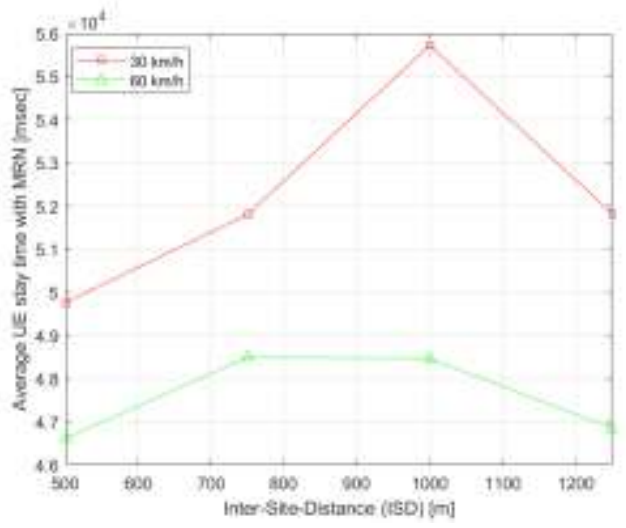

Fig. 11. Average UE stay time with MRN.

by $4.5 \%$ on average for all simulated cases of Case 2 in comparison to Case 1 .

Fig. 10 shows the average number of UEs (twenty-six UEs to twenty-eight UEs) connected with the MRN during the simulation. It has to be noted that we only simulate a cluster of twenty-four UEs traveling on the bus but Fig. 10 shows that the minimum number of connected UEs with the MRN is twentysix. This means that some of the outboard UEs moving nearby the MRN are connected with the MRN. Fig. 11 illustrates the average UE stay time with the MRN that varies from around 46 $\mathrm{sec}$ to $56 \mathrm{sec}$. But our simulation time is $60 \mathrm{sec}$. This means that the average UE stay time is less because of the connection of outboard UEs with the MRN we noted in Fig. 10. The connection of outboard UEs with MRN may be lost after a few seconds, causes a reduction in average UE stay time with MRN. This also reduces the quality of experience (QoE) of the outboard UEs moving near the MRN. It is clear from the graph that the low bus speed has the highest UE stay time with the MRN that is expected. The graph also shows that the low ISD has the lowest average UE stay time due to less number of an average outboard UEs connected to the MRN with their short stay time (see Fig. 10, i.e. for ISD $500 \mathrm{~m}$ and speed $60 \mathrm{~km} / \mathrm{h}$ case, onboard UEs $=24$, outboard UEs $=2$ ). Then, it increases as the number of outboard UEs increase and their stay time with the MRN increase due to large cell sizes with low coverage along the cell edges, especially at low speed. After that, it starts decreasing again for very high ISDs because of the high PP rate, we noted in Fig. 6. We can argue that a high PP rate reduces the stay time of the outboard UEs with the MRN.

\section{CONCLUSIONS}

In this paper, a simulation analysis of the $\mathrm{HO}$ performance and power consumption improvements is presented, when a MRN is installed at the roof-top of a bus traveling along the celledge of the DeNBs. High improvements in terms of $\mathrm{HO}$ rates, HOFR, PP rates, and power consumption are observed for the scenario where MRN performs the HO procedure to the DeNBs. Such improvements come at the cost of insignificant improvement in service interruption time (only $4.5 \%$ improvement on average for all simulated cases) due to the high HOFR of the MRN. The dominant single point of failure cases of the MRN is due to RLC HO confirmation transmission errors. We also found that the average number of UEs connected to the MRN is higher than the number of the benefiting UEs traveling on the bus, because of the connection of outboard UEs moving nearby the MRN. In the future, we will prevent the non-bus UEs connection with the MRN so as to improve the QoE of the nonbus users moving nearby the bus. Also, we will target at reducing the MRN HO failure cases and the power consumption, especially by addressing the adequacy of UL based mobility.

\section{REFERENCES}

[1] A. L. Yusof, B. A. Bakar, N. Yaacob, and M. A. Zainali, "Mobile Relay Handover Procedure in Train For 4G LTE-Advanced Network" Journal of Telecommunication, Electronic, and Computer Engineering, vol. 9, No. 22, pp. 49-53, 2017.

[2] L. Chen, et. al, "Mobile Relay in LTE-Advanced Systems" IEEE Communications Magazine, pp. 144-151, Nov 2013.

[3] 3GPP TR 36.836, "Study on mobile relay for Evolved Universal Terrestrial Radio Access (E-UTRA); (Release 12), June 2014.

[4] Yangyang Chen. "Performance analysis of mobile relays for LTE", Networking and Internet Architecture [cs.NI]. Télécom Bretagne; Université de Rennes 1, 2015.

[5] M. Tayyab, G.P. Koudouridis, X. Gelabert "A Simulation Study on LTE Handover and the Impact of Cell Size". Broadband Communications, Networks, and Systems. (BROADNETS 2018).

[6] A. Krendzel, "LTE-A Mobile Relay Handling: Architecture Aspects", European Wireless, April 2013.

[7] 3GPP TS 36.300, “(E-UTRA) and (E-UTRAN); Overall description; Stage 2 (Release 15)" V15.0.0, Section 10, pp. 93-143, Dec. 2017.

[8] 3GPP TR 36.814, "Further advancements for E-UTRA physical layer aspects (Release 9)," V9.0.0, Mar 2010.

[9] 3GPP TS 36.331, "E-UTRA Radio Resource Control (RRC); Protocol specification (Release 9)", v9.2.0, Mar 2010.

[10] 3GPP TS 36.133, "Requirements for support of radio resource management (Release 9)," v9.15.0, Mar 2013.

[11] M. Tayyab, G. P. Koudouridis, X. Gelabert and R. Jäntti, "Signaling Overhead and Power Consumption during Handover in LTE", IEEE Wireless Communications and Networking Conference (WCNC), 2019.

[12] M. Tayyab, G. P. Koudouridis, X. Gelabert and R. Jäntti, "Receiver Power Consumption during Handover in LTE", IEEE 5G world forum conference, pp. 74-79, 2019.

[13] M. Tayyab, X. Gelabert and R. Jäntti, “A Survey on Handover Management: From LTE to NR", IEEE Access Journal, Vol. 7, Issue 1, pp. 118907-118930, August 2019.

[14] J. Rodriguez et al., "SECRET - Secure Network Coding for Reduced Energy Next Generation Mobile Small cells", IEEE Internet Technologies and Applications (ITA) conference, pp. 329-333, 2017. 